\title{
Spinal cord bypass surgery using peripheral nerve transfers: review of translational studies and a case report on its use following complete spinal cord injury in a human
}

\author{
Experimental article \\ JefFrey S. Oppenheim, M.D., ${ }^{1}$ DANiel E. Spitzer, M.D., ${ }^{1}$
AND Christopher J. Winfree, M.D., F.A.C.S.
}

${ }^{1}$ Section of Neurological Surgery, Good Samaritan Hospital, Suffern; and ${ }^{2}$ Department of Neurological Surgery, Columbia University Medical Center, New York, New York

\begin{abstract}
Spinal cord injury has been studied in a variety of in vitro and in vivo animal models. One promising therapeutic approach involves the transfer of peripheral nerves originating above the level of injury into the spinal cord below the level of injury. A model of spinal cord injury in rodents has shown the growth of peripheral nerve fibers into the spinal cord, with the subsequent development of functional synaptic connections and limb movement. The authors of this paper are currently developing a similar model in felines to assess the cortical control of these novel repair pathways. In an effort to determine whether these neurotization techniques could translate to spinal cord injury in humans, the authors treated a patient by using intercostal nerve transfer following complete acute spinal cord injury. The case presented details a patient with paraplegia who regained partial motor and sensory activity following the transfer of intercostal nerves, originating above the level of the spinal cord injury, into the spinal canal below the level of injury. The patient recovered some of his motor and sensory function. Notably, his recovered hip flexion showed respiratory variation. This finding raises the possibility that intercostal nerve transfers may augment neurological recovery after complete spinal cord injury. (DOI: 10.3171/FOC.2009.26.2.E6)
\end{abstract}

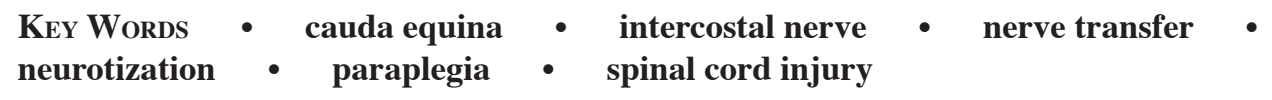

$\mathrm{E}$ STIMATES of the incidence of spinal cord injuries vary from 30 to 40 new cases per million persons annually in the US, and the average age of the victim of spinal cord injury is $<40$ years. ${ }^{29}$ Approximately one-quarter of these injuries result in complete paraplegia. ${ }^{18,29} \mathrm{~A}$ simple technique that facilitates the recovery of some lower-extremity motor and sensory activity after a complete paraplegic spinal cord injury would have tremendous value. The rehabilitation potential, cost of care, risk of complications and/or death, and quality of life could all improve on recovery from a complete to partial spinal cord injury.

Neurotization, or nerve transfer, is most commonly used to treat brachial plexus injury by connecting a donor nerve, such as the spinal accessory or intercostal nerves, to a denervated peripheral nerve target. ${ }^{7,28}$ After the transfer, axons grow from the donor nerve into the recipient

Abbreviation used in this paper: MRC = Medical Research Council. nerve, innervating previously denervated muscles. For example, nerve transfers can permit recovery of shoulder abduction and arm flexion even in a flail arm.

In experimental studies, researchers have used nerve transfers to bypass spinal cord injury. In brief, an intercostal nerve originating above the level of injury is separated from its target muscle and transferred to nerve roots or into the spinal cord below the level of spinal cord injury. In an effort to explore the potential of this strategy in the clinical setting, we performed an intercostal nerve transfer into the spinal canal below the level of injury in a patient undergoing surgery for treatment of a complete spinal cord injury.

\section{Illustrative Case}

History and Examination. This 48-year-old man sustained an injury as the result of a fall from a ladder. Motor and sensory examinations were consistent with a complete T-11 level spinal cord injury. Spinal CT re- 


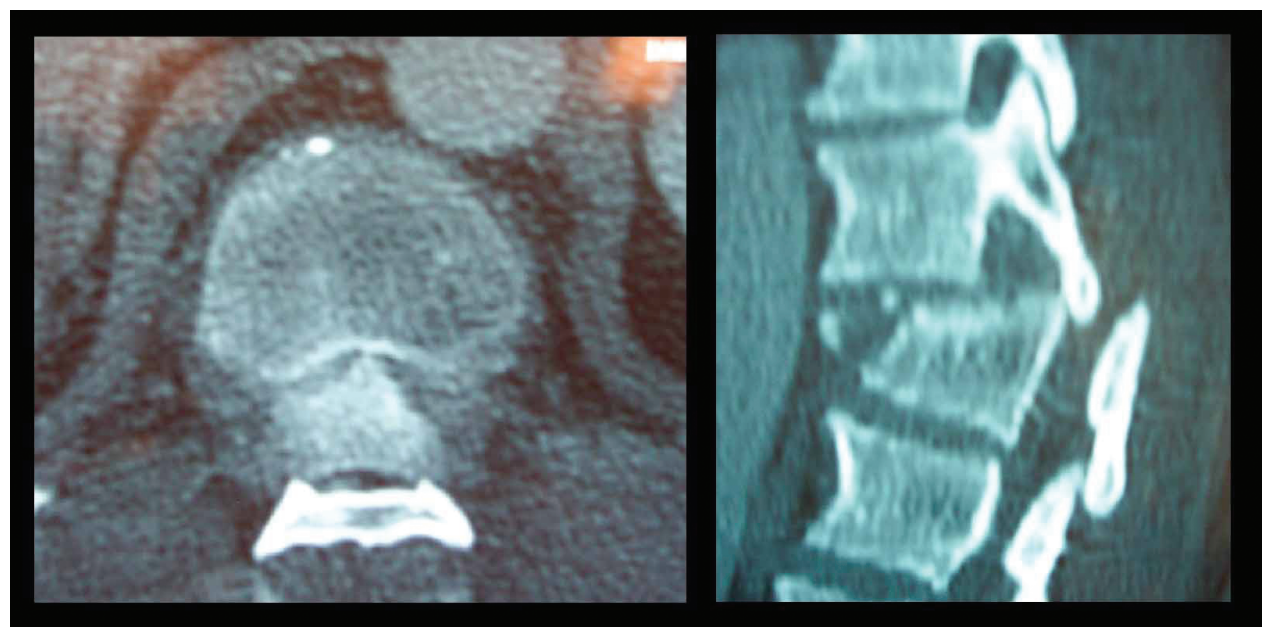

FIG. 1. Axial (left) and sagittal (right) reconstruction CT scans clearly demonstrating the anterolisthesis of T-11 on T-12, loss of height of the anterior T-12 vertebral body, osseous obliteration of the spinal canal, and disruption of the T11-12 posterior elements.

vealed a T11-12 fracture dislocation with complete canal compromise (Fig. 1).

Operation. Forty-eight hours after injury, the patient underwent a T11-12 laminectomy and a T-9 to L-1 posterior instrumented fusion. Before implanting the instrumentation, the T-11 nerve roots were identified and dissected $\sim 2 \mathrm{~cm}$ distal from the canal. A partial costotransversectomy was performed bilaterally to unroof these roots. The origin of the T-11 roots was visualized just above the level of the cord injury. The roots were cut distally and transferred into an elliptical incision made into the dura of the spinal cord just below the level of the injury. The implantation was angled so that the roots would point inferiorly

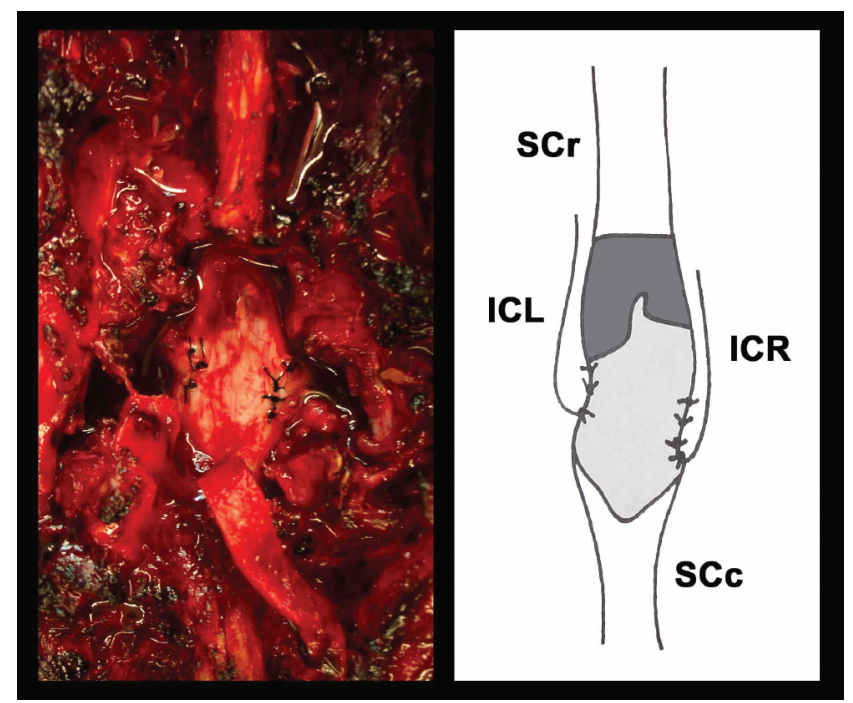

FIG. 2. Intraoperative photograph (left) obtained after a T11-12 laminectomy and a schematic (right) showing implantation of the left (ICL) and right (ICR) T-11 intercostal nerves through the dura (light gray) into the spinal canal just caudal to the level of spinal cord injury (dark gray). The top of the schematic illustrates the rostral spinal cord (SCr), whereas the bottom shows the caudal spinal cord (SCc), partially obscured by a cottonoid. into the canal (Fig. 2). No attempt was made to directly attach the roots or fascicles to either the distal spinal cord or any of the roots of the cauda equina.

Postoperative Course. Postoperatively, the patient remained paraplegic with an unchanged sensory level and a neurogenic bladder. He subsequently spent 8 weeks at an inpatient rehabilitation unit where he was taught to selfcatheterize and use a bowel regimen. Urinary tract infections and a deep venous thrombosis complicated his clinical course. At 5 months postoperatively, the patient began to report a vague awareness of sensation in his thighs. Sensation below the knees was absent. No motor function in the lower extremities was observed. At 10 months postoperatively, the patient began to demonstrate recovery of hip adduction (MRC Grade 2/5) and flexion (MRC Grade $2 / 5$ ). The patient noted that he could induce more powerful adduction by exhaling firmly. Spontaneous movement of his legs was observed in cycle with respirations. No clinical improvement in urinary or bowel function was reported.

\section{Discussion}

\section{The Spinal Cord Injury Problem}

Primary spinal cord injury results from the physical disruption of the spinal cord structure at the time of initial injury due to penetrating objects, bone fragments, hematoma, or other mechanisms. ${ }^{17}$ Secondary injury represents additional damage that occurs in a delayed fashion in the tissues surrounding the primary injury. ${ }^{32}$ Tissue ischemia, excitotoxicity, inflammation, apoptosis, and free radical injury are all mechanisms contributing to secondary injury. Unfortunately, after injury the CNS neurons display little tendency for regeneration; ${ }^{34}$ and even if they did, the extracellular matrix is laden with growth-inhibition substances that restrict nerve cell regeneration..$^{12}$ Moreover, the site of spinal cord injury is typically replaced with a dense glial scar, providing a mechanical barrier against regenerative processes. ${ }^{31}$ One strategy to treat spinal cord 
injury is to bypass the site of injury with functional nerve bridges.

\section{Spinal Cord Bypass: Animal Studies}

Early efforts to supply innervation to more caudal structures below a level of injury date back to 1907 when lumbar nerve roots were anastomosed to the ventral sacral roots in dogs. Stimulation of the transferred roots produced bladder contraction and micturition. ${ }^{19}$ Similar studies decades later involved the use of related strategies to enable the recovery of lower-extremity function in $\operatorname{dog} \mathrm{s}^{14}$ and erectile function in baboons. ${ }^{15}$ In an experiment in cats, the researchers used an L7-S1 peripheral nerve root transfer to recover bladder function. 6,35 After an appropriate period of recovery, intravesical pressures showed respiratory variation, stimulation of the transferred nerves led to bladder contraction, sectioning of the transferred nerves abolished this function, and histological analysis confirmed growth of the transferred axons down the target nerves.

Intercostal nerves were chosen as the donor nerves for transfer to the lumbar roots in canine experiments. ${ }^{26}$ Following a T-11 transfer to L-6, some animals were eventually able to ambulate without a limp. A histological follow-up analysis in these animals revealed axon growth from the donor to the target nerves as well as morphological changes in the muscle consistent with denervation and subsequent reinnervation. ${ }^{33}$ Later studies in rabbits ${ }^{10}$ and $\operatorname{dog} \mathrm{s}^{37}$ demonstrated the feasibility of transferring multiple intercostal nerves to a single lumbar nerve root and to multiple lumbar roots in rats. ${ }^{42} \mathrm{~A}$ second study showed partial recovery of limb function following the transfer of multiple intercostal nerves to multiple lumbar nerve roots in the rat. ${ }^{24}$

A variant of a spinal cord bypass without using nerve transfers has been developed in rats ${ }^{23,24}$ and later in primates. ${ }^{20,21}$ In this model, the proximal terminus of peripheral nerve autografts is implanted into the ventral horn of the spinal cord below the level of injury, whereas the distal ends are anastomosed to the lumbar ventral nerve roots. Data from this model have suggested that anterior horn motor neurons send recovering axons down the nerve grafts to reinnervate the distal lumbar nerve-supplied targets.

\section{Spinal Cord Bypass: Human Cadaver Studies}

A feasibility study in human cadavers has shown that the T-11 intercostal nerve can be readily transferred to the L-5 nerve root in both infants and adults. ${ }^{26}$ Intercostal nerves have been reported to be acceptable donor nerves for transfer to the sacral nerve roots at an intraspinal level in a cadaver study. ${ }^{40} \mathrm{~A}$ more extensive set of studies in adult cadavers has revealed that the T9-11 intercostal nerves can be sufficiently harvested and transferred to targets within the cauda equina. , $^{8,38,39}$

\section{Spinal Cord Bypass: Human Clinical Studies}

The first description of a nerve transfer to treat a human following a spinal cord injury dates back to 1912. In that case report, a patient who had sustained an L-2 level injury underwent transfer of 1 of the extradural L-1 nerve roots to the S-3 and S-4 roots within the cauda equina. ${ }^{13}$ The patient apparently regained some functional bladder control 8 months later. There is a brief description of another series of patients with high lumbar spinal column injuries who underwent intercostal to lumbar nerve root anastomosis. ${ }^{27}$ Of the 8 patients in the series, only 1 showed improvement, but few details of either the surgical technique or the patient characteristics were described. In an early case report, ${ }^{4}$ a child with myelomeningocele and a neurogenic bladder was treated with neural plate excision and anastomosis of 1 pair of thoracic intercostal nerves to 2 pairs of sacral nerve roots. Although the bladder was initially atonic, by 8 months the patient recovered reflex micturition. A second report by the same group of researchers ${ }^{5}$ details the treatment of 2 patients with complete L-1 spinal cord injury following T12-L1 fractures. In both patients, the bilateral T-12 intercostal nerves were transferred to the bilateral S2-3 nerves below the level of injury within 2 weeks of injury. Both patients recovered some sacral sensation and voluntary voiding and had low postvoid residual volumes.

Two patients with myelomeningocele underwent T10-11 intercostal nerve transfer to the lumbosacral nerve roots distal to the congenital lesion. ${ }^{11}$ Although minimal follow-up data were reported, both patients apparently experienced reinnervation of select lower-extremity muscles, as noted on electrodiagnostic studies and clinical examination. Another adult patient suffered a traumatic L1-2 fracture, resulting in a complete spinal cord injury at the T-12 level..$^{30}$ The bilateral T8-9 intercostal nerves were transferred to the bilateral L-2 and (presumably) S-1 nerve roots. Although this report showed the feasibility of this technique, no follow-up data were included.

A reasonably large series of patients with spinal cord injury underwent 1 of 2 types of nerve transfer surgery. ${ }^{9}$ In the first treatment group, several pairs of lower intercostal nerves were anastomosed to the lumbosacral nerve roots within the cauda equina. In the second group, several pairs of intercostal nerves were anastomosed to the L2-4 nerve roots in the retroperitoneal lumbar plexus. Although most of these patients had sustained a complete spinal cord injury, many recovered some, albeit modest, motor function. Specifically, 5 of the 21 patients in the study experienced recovery of lower-extremity muscle power ranging from MRC Grade 1 to 3. Another series of patients ${ }^{41}$ who had suffered low thoracic spinal cord injury-all but 1 with complete injury-underwent transfer of the 2-4 intercostal nerves above the level of injury to the L1-4 nerve roots within the spinal canal below the level of injury. More than three-quarters of the patients recovered sufficient lower-extremity motor function to permit ambulation with an assistive device. Nerve transfers were used in a series of 11 patients following L-1 complete spinal cord injuries. ${ }^{25}$ One year after transferring the bilateral T11-12 intercostal nerves to the bilateral S2-3 nerve roots, patients had significant improvements in bladder function, sacral sensations, and sacral reflexes.

The group that created the model of spinal cord bypass in which peripheral nerve autografts are implanted proximally into the ventral spinal cord rostral to the level 
of injury and distally into the ventral lumbar nerve roots attempted this technique in a human. ${ }^{36}$ This patient had sustained a complete T-9 level spinal cord injury 3 years before the bypass surgery. He received peripheral nerve autografts, which were inserted into the T7-8 ventral spinal cord proximally and anastomosed to the L2-4 lumbar roots within the spinal canal distally. Several months postoperatively, the patient recovered modest thigh adduction and knee extension on clinical and electrodiagnostic examination.

\section{A New Approach to Spinal Cord Bypass}

The transfer of peripheral nerves originating above the level of spinal cord injury to peripheral nerve targets below the level of injury has limitations. For example, the axons must grow from the site of anastomosis to the target muscles, a process that may require years, depending on the length of the target nerve. Moreover, the availability of donor axons is limited, thus hindering the degree to which 1 or more targets can be reinnervated.

If the peripheral nerve donors were transferred directly into the spinal cord, then they could potentially tap into existing spinal cord circuits below the level of injury. ${ }^{1}$ A relatively small donor nerve could exert control over a fairly complex set of circuits, without the need for extensive fiber growth into the periphery, given that those pathways are already in place. In the literature there is an early report of intercostal transfer to the spinal cord. ${ }^{16}$ An adult male patient had sustained a gunshot wound to the spinal cord, resulting in complete transection at about the T-11 level. Four months later he underwent a bilateral T-8 and T-10 intercostal nerve harvest. One pair of intercostal nerves was transferred to clumps of sacral nerve roots, whereas the second pair was inserted into the substance of the conus medullaris via midline stab incisions. Unfortunately, the patient died before the acquisition of meaningful follow-up data. However, postmortem findings did demonstrate axonal growth into the recipient sacral nerves past the anastomoses and, notably, into the substance of the conus.

This model of spinal cord bypass surgery recently has been investigated in a rodent model. ${ }^{2}$ An intercostal nerve was transferred into the spinal cord caudal to an ipsilateral hemisection. Peripheral nerve fibers grew into the ventral horn of the spinal cord and made functional synapses on alpha motor neurons. ${ }^{2,3}$ Stimulation of the transferred nerve generated visible contractions in the paralyzed lower extremity. Furthermore, this strategy partially ameliorated the spasticity that results following cord hemisection. ${ }^{2}$

In the current report, we describe a patient with a complete spinal cord injury who underwent bilateral transfer of the T-11 nerves into the spinal canal below the level of injury. We chose to insert the transferred intercostal nerves into the spinal canal but not into the spinal cord itself, to avoid causing further cord injury. Thus, this technique differs quite a bit from that in the rodent model described above. ${ }^{2}$ A strategy involving the direct insertion of nerves into the spinal cord would be appropriate only in the setting of chronic spinal cord injury in which the level of recovery is absent and stable below the level of injury. It is our opinion that the incisions needed to insert the nerves into the cord may cause unacceptable damage to a partially injured spinal cord in the acute setting, and thus compromising its ability to recover.

Nevertheless, in the illustrative case herein described, the patient recovered some hip flexion. Curiously, the muscle movements were associated with respiratory variation, which would be expected if under control by the intercostal (respiratory) nerves. Additionally, electromyographic studies of the hip flexor muscles showed characteristic respiratory variation. Although there was no way to know for certain that this patient was not going to recover some of this function anyway, the respiratory variation in the recovered muscles suggests that a portion of this function is controlled by the intercostal nerves.

Why peripheral axons grow into the CNS so well, whereas central axons do not, is a mystery but likely stems from phenotypic differences between the 2 types of fibers. Stimulation of the transferred peripheral nerve results in limb movement when sufficient recovery time has elapsed in a rodent model..$^{2,3}$ Intermittent stimulation produces intermittent leg movement through these novel spinal cord synapses, not through another pathway, such as via the spinal nerve roots. There is no evidence to suggest that the growth of a peripheral nerve directly into the spinal roots even occurs. In fact, such a pathway would be anatomically impossible in the animal models, because the transferred axons would have no way of entering the intact spinal nerve roots. Similarly, it would be difficult to imagine a feasible pathway for the peripheral axons from the transferred nerves in our patient to find their way for a couple of segments caudally and to enter otherwise undamaged spinal nerve roots to exert their effects.

Our patient gained leg movement with respiratory muscle contraction. Sectioning the peripheral nerve bridge in animal models abolishes limb movement. ${ }^{2,3} \mathrm{We}$ suspect that a similar result would occur if our patient underwent an appropriate intercostal nerve block. Unfortunately, in the absence of any reasonable expectation of a clinical benefit, this procedure would be unethical, and we have not advocated it.

Obviously, there is no way to prove that our patient's recovery was due solely to the nerve transfer, as opposed to some recovery of intrinsic spinal cord function. Nevertheless, the presence of respiratory variation in the muscle contractions is certainly suggestive of at least a contribution by the transferred intercostal circuitry. This problem will always be encountered when studying this population of patients. Thus, within a population of spinal cord-injured patients, there would be no way to reliably determine if the outcome for any given patient is due to spontaneous recovery or to the treatment itself. We advocate the exploration of this problem in a larger clinical study so that meaningful differences between treatment groups can be revealed if present.

\section{Conclusions}

A spinal cord bypass utilizing intercostal nerve transfers to spinal cord targets caudal to the level of injury may represent a viable approach in treating complete spinal 
cord injury in humans. Future animal studies and subsequent clinical trials in humans will, we hope, delineate the utility of this treatment strategy.

\section{Disclaimer}

The authors report no conflict of interest concerning the materials or methods used in this study or the findings specified in this paper.

\section{References}

1. Barbeau H, McCrea DA, O’Donovan MJ, Rossignol S, Grill WM, Lemay MA: Tapping into spinal circuits to restore motor function. Brain Res Brain Res Rev 30:27-51, 1999

2. Campos L, Meng Z, Hu G, Chiu DTW, Ambron RT, Martin JH: Engineering novel spinal circuits to promote recovery after spinal cord injury. J Neurosci 24:2090-2101, 2004

3. Campos LW, Chakrabarty S, Haque R, Martin JH: Regenerating motor bridge axons refine connections and synapse on lumbar motoneurons to bypass chronic spinal cord injury. J Comp Neurol 506:838-850, 2008

4. Carlsson CA, Sundin T: Forefront: preliminary report. Reconstruction of efferent pathways to the urinary bladder in a paraplegic child. Rev Surg 24:73-76, 1967

5. Carlsson CA, Sundin T: Reconstruction of afferent and efferent nervous pathways to the urinary bladder in two paraplegic patients. Spine 5:37-41, 1980

6. Carlsson CA, Sundin T: Reconstruction of severed ventral roots innervating the urinary bladder. An experimental study in cats. Scand J Urol Nephrol 2:199-210, 1968

7. Chuang DC: Neurotization procedures for brachial plexus injuries. Hand Clin 11:633-645, 1995

8. Court C, Vialle R, Lepeintre JF, Tadie M: The thoracoabdominal intercostal nerves: an anatomical study for their use in neurotization. Surg Radiol Anat 27:8-14, 2005

9. Dai KR, Yu CT, Wu RS, Zhang XF, Yuan JX, Sun YH: Intercostal-lumbar-spinal nerve anastomoses for cord transection. A preliminary investigation. J Reconstr Microsurg 1:223226, 1985

10. de Divitiis E, Donzelli R, Caputi F, Crisci C, Gargiulo G, Francica D: Experimental model of nervous anastomosis between intercostal and lumbar nerves in the rabbit. J Neurosurg Sci 28:153-156, 1984

11. Epstein F, Spielholz N, Battista A, McCarthy J: Delayed cauda equina reconstruction in meningomyelocele: preliminary report. Neurosurgery 6:540-541, 1980

12. Filbin MT: Myelin-associated inhibitors of axonal regeneration in the adult mammalian CNS. Nat Rev Neurosci 4:703713,2003

13. Frazier $\mathrm{CH}$, Mills $\mathrm{CK}$ : Intradural root anastomosis for the relief of paralysis of the bladder. JAMA 59:2202-2206, 1912

14. Freeman LW: Functional regeneration of spinal nerve roots. $\mathbf{Q}$ Bull Indiana Univ Med Cent 11:43-46, 1949

15. Freeman LW: Neuronal regeneration in the central nervous system of man. Successful growth of intercostal-spinal nerve anastomosis and growth of intercostal nerve-spinal cord implant. J Neurosurg 18:417-422, 1961

16. Freeman LW: Observations on spinal nerve root transplantation in the male guinea baboon. Ann Surg 136:206-210, 1952

17. Hulsebosch CE: Recent advances in pathophysiology and treatment of spinal cord injury. Adv Physiol Educ 26:238255,2002

18. Jackson AB, Dijkers M, DeVivo M, Poczatek R: A demographic profile of new traumatic spinal cord injuries: change and stability over 30 years. Arch Phys Med Rehabil 85:17401748,2004

19. Kilvington B: An investigation on the regeneration of nerves, with regard to surgical treatment of certain paralysis. BMJ 1:988-990, 1907

20. Liu S, Aghakhani N, Boisset N, Said G, Tadie M: Innervation of the caudal denervated ventral roots and their target muscles by the rostral spinal motoneurons after implanting a nerve autograft in spinal cord-injured adult marmosets. J Neurosurg 94 (1 Suppl):82-90, 2001

21. Liu S, Bodjarian N, Langlois O, Bonnard AS, Boisset N, Peulve $\mathrm{P}$, et al: Axonal regrowth through a collagen guidance channel bridging spinal cord to the avulsed C6 roots: functional recovery in primates with brachial plexus injury. J Neurosci Res 51:723-734, 1998

22. Liu S, Damhieu P, Devanze P, Said G, Heard JM, Tadie M: Efficient reinnervation of hindlimb muscles by thoracic motor neurons after nerve cross-anastomosis in rats. J Neurosurg 99:879-885, 2003

23. Liu S, Kadi K, Boisset N, Lacroix C, Said G, Tadie M: Reinnervation of denervated lumbar ventral roots and their target muscle by thoracic spinal motoneurons via an implanted nerve autograft in adult rats after spinal cord injury. J Neurosci Res 56:506-517, 1999

24. Liu S, Peulve P, Jin O, Boisset N, Tiollier J, Said G, et al: Axonal regrowth through collagen tubes bridging the spinal cord to nerve roots. J Neurosci Res 49:425-432, 1997

25. Livshits A, Catz A, Folman Y, Witz M, Livshits V, Baskov A, et al: Reinnervation of the neurogenic bladder in the late period of the spinal cord trauma. Spinal Cord 42:211-217, 2004

26. Malik HG, Buhr AJ: Intercostal nerve transfer to lumbar nerve roots. Part I: development of an animal model and cadaver studies. Spine 4:410-415, 1979

27. Makino H, Takamura R, Yamano N, Takahashi H, Owada M, Izumi K, et al: Clinical experience on intercostal and cauda equina motoric nerve anastomosis for paraplegia. Neurol Med Chir (Tokyo) 6:146-147, 1964

28. Midha R: Nerve transfers for severe brachial plexus injuries: a review. Neurosurg Focus 16(6):1-10, 2004

29. Nobunaga AI, Go BK, Karunas RB: Recent demographic and injury trends in people served by the model spinal cord injury care systems. Arch Phys Med Rehabil 80:1372-1382, 1999

30. Patil A: Intercostal nerves to spinal nerve roots anastomosis (spinal cord bypass) and Harrington rod fusion in traumatic paraplegia-technical note. Acta Neurochir (Wien) 57:299303, 1981

31. Qiu J: Glial inhibition of nerve regeneration in the mature mammalian CNS. Glia 29:166-174, 2000

32. Ramer LM, Ramer MS, Steeves JD: Setting the stage for functional repair of spinal cord injuries: a cast of thousands. Spinal Cord 43:134-161, 2005

33. Sangalang VE, Buhr AJ, Malik HG: Intercostal nerve transfer to lumbar nerve roots. Part II: neuropathologic findings in the animal model. Spine 4:416-422, 1979

34. Schwab ME: Repairing the injured spinal cord. Science 295:1029-1031, 2002

35. Sundin T, Carlsson CA: Reconstruction of severed ventral roots innervating the urinary bladder. An experimental study in cats II. Regeneration studies. Scand J Urol Nephrol 6:185196, 1972

36. Tadie M, Liu S, Robert R, Guiheneuc P, Pereon Y, PerrouinVerbe B, et al: Partial return of motor function in paralyzed legs after surgical bypass of the lesion site by nerve autografts three years after spinal cord injury. J Neurotrauma 19:909916, 2002

37. Tok S, Schmid UD, Ferbert A, Davenport T: Intercostolumbar spinal nerve anastomosis. An experimental study in dogs. Spine 16:463-466, 1991

38. Vialle R, Court C, Harding I, Lepeintre JF, Khouri N, Tadie M: Multiple lumbar plexus neurotizations of the ninth, tenth, and eleventh intercostal nerves. Clin Anat 19:51-58, 2006 


\section{J. S. Oppenheim, D. E. Spitzer, and C. J. Winfree}

39. Vialle R, Lepeintre JF, Court C, Loureiro MC, Lacroix C, Tadie M: Anatomic feasibility of using the ninth, $10^{\text {th }}$, and $11^{\text {th }}$ intercostal nerves for the treatment of neurological deficits after damage to the spinal cord. J Neurosurg Spine 4:225-232, 2006

40. Vorstman B, Schlossberg S, Landy S, Kass L: Nerve crossover techniques for urinary bladder reinnervation: animal and human cadaver studies. J Urol 137:1043-1047, 1987

41. Zhang S, Johnston L, Zhang Z, Ma Y, Hu Y, Wang J, et al: Restoration of stepping-forward and ambulatory function in patients with paraplegia: rerouting of vascularized intercos- tal nerves to lumbar nerve roots using selected interfascicular anastomosis. Surg Technol Int 11:244-248, 2003

42. Zhao S, Beuerman RW, Kline DG: Neurotization of motor nerves innervating the lower extremity by utilizing the lower intercostal nerves. J Reconstr Microsurg 13:39-45, 1997

Manuscript submitted October 15, 2008.

Accepted December 12, 2008.

Address correspondence to: Christopher J. Winfree, M.D., Department of Neurological Surgery, 710 West 168th Street, 5th Floor, New York, New York 10032. email: cjw12@columbia.edu. 